\title{
A mobile VR-based respiratory biofeedback game to foster diaphragmatic breathing
}

\author{
Christoph Rockstroh ${ }^{1}$ · Johannes Blum ${ }^{1}$ · Anja S. Göritz ${ }^{1}$
}

Received: 5 May 2020 / Accepted: 16 September 2020 / Published online: 5 October 2020

(c) The Author(s) 2020

\begin{abstract}
Virtual reality (VR) has become popular in mental health research. Several studies have explored the use of VR in the context of biofeedback protocols. In the present paper, we report on the development and evaluation of a VR-based respiratory biofeedback game to foster diaphragmatic breathing. The game integrates respiratory biofeedback, restorative VR and gamification. The game is designed to run on a mobile, all-in-one VR headset. Notably, an integrated VR hand controller is utilized as a sensor to detect respiration-induced movements of the diaphragm. In a longitudinal within-subjects study, we explored the feasibility of the game and tested the effectiveness of six training sessions. Participants reported a pleasant user experience. Moreover, the results show that the brief VR-based breathing training increased perceived breath awareness, improved diaphragmatic breathing, increased relaxation, decreased perceived stress, reduced symptoms of burnout and boosted relaxation-related self-efficacy. Future studies need to address the generalizability and long-term stability of the results, compare the approach with existing treatments and fine-tune the training components.
\end{abstract}

Keywords Virtual reality $\cdot$ Respiratory biofeedback $\cdot$ Diaphragmatic breathing $\cdot$ Serious game $\cdot$ Stress reduction $\cdot$ Selfefficacy

\section{Introduction}

\subsection{Virtual reality in mental health research}

Virtual reality (VR) is a powerful tool for mental health interventions. Contemporary head-mounted VR headsets with six-degrees-of-freedom head and hand tracking provide a deep level of immersion. Thus, customizable virtual worlds can mimic real environments and benefit from comparable effects. Researchers and practitioners

Christoph Rockstroh and Johannes Blum contributed equally and share first authorship.

Christoph Rockstroh

christoph.rockstroh@psychologie.uni-freiburg.de

Johannes Blum

johannes.blum@psychologie.uni-freiburg.de

Anja S. Göritz

goeritz@psychologie.uni-freiburg.de

1 Department of Occupational and Consumer Psychology, Albert-Ludwigs-Universität Freiburg, Engelbergerstr. 41, 79106 Freiburg, Germany alike can create tailored restorative virtual experiences to tackle stress-related mental health problems. While VR has technically been around for decades, the recent technological improvements such as reduced latency, higher tracking accuracy, better displays, increased ease of use and decreased cost have sparked researchers' interest. A substantial body of research has investigated the feasibility and effectiveness of VR in several mental health contexts. For instance, VR was used to create immersive experiences for exposure therapy (Carl et al. 2019), pain management (Mallari et al. 2019; Scapin et al. 2018), mental wellness (Roche et al. 2019) or mindfulness and meditation training (Chandrasiri et al. 2020; Navarro-Haro et al. 2017).

\subsection{Virtual reality biofeedback}

In digital mental health, several studies explored the use of VR in the context of biofeedback protocols. In biofeedback, physiological signals are detected by sensors and fed back to the user in realtime to provide information about otherwise hardly observable bodily functions with the aim of improving the user's conscious control (for reviews, see Giggins et al. 2013; Yu et al. 2018). The user may 
learn to voluntarily control their electrodermal activity, heart rate variability, brain activity or respiration, among others. Recently, VR has been combined and tested with biofeedback of electroencephalographic oscillations (e.g., Berger and Davelaar 2018; Kosunen et al. 2016), electrodermal activity (e.g., Gromala et al. 2015; Hølledig and Petersen 2018; Rockstroh et al. 2020), heart rate (e.g., Gradl et al. 2018; Houzangbe et al. 2019) and heart rate variability (e.g., Rockstroh et al. 2019; Blum et al. 2019).

A straightforward biofeedback approach for stress reduction is respiratory biofeedback, which entails the measurement and feedback of physiological parameters related to respiration (e.g., direct inhalation or exhalation actions, respiratory rate) to heighten the awareness of the breath as well as to foster a desired breathing style (e.g., slow diaphragmatic breathing). Performing diaphragmatic breathing is a widespread feature in stress reduction approaches as it offers physiological as well as psychological benefits. Slow diaphragmatic breathing modulates vagal tone of the parasympathetic nervous system by maximizing the respiratory sinus arrhythmia (Vaschillo et al. 2006; Yasuma and Hayano 2004). Thereby, slow regular diaphragmatic breathing can trigger an autonomic relaxation response (Subbalakshmi et al. 2014) and helps regain the balance of the autonomic nervous system. Consequently, diaphragmatic breathing is used in evidence-based exercises to reduce anxiety and negative affect (e.g., Chen et al. 2017; Ma et al. 2017) as well as improve mood and relaxation (e.g., Hopper et al. 2019; Perciavalle et al. 2017). Respiratory biofeedback is meant to increase the awareness toward the breath, help the trainees evaluate their current breathing style to improve it if necessary and offer reinforcement learning by providing pleasing and rewarding feedback stimuli (cf. Gaume et al. 2016; Sherlin et al. 2011). Respiratory biofeedback has also been combined with VR (e.g., Sra et al. 2018; Tinga et al. 2019; van Rooij et al. 2016).

VR offers many benefits when combined with biofeedback. For instance, VR can increase the visual appeal and salience of the feedback stimuli, which can foster motivation and engagement (Rockstroh et al. 2019; Soyka et al. 2016; van Rooij et al. 2016). Moreover, the virtual environment is fully controllable and customizable to offer a safe and supportive training environment (e.g., relaxing and comforting environments; Blum et al. 2019; Gromala et al. 2015; van Rooij et al. 2016). Additionally, blocking unwanted external audiovisual stimuli and replacing them with desired and task-relevant stimuli reduces distraction and supports a sustained focus on the biofeedback task (Blum et al. 2019). Lastly, biofeedback can increase the trainees' belief in their own ability to relax both body and mind (i.e., relaxation-related self-efficacy) because success in exhibiting a healthy target behavior or skill is fed back in real time and thus immediately observable (cf. Giardino et al. 2004; Paul and Garg 2012; Teufel et al. 2013). When combined with immersive VR, those self-efficacy-inducing feedback stimuli become more vivid, which can boost the effect of the biofeedback training on relaxation-related selfefficacy (Blum et al. 2019; Weerdmeester et al. 2017).

To date, most VR biofeedback implementations require a considerable amount of effort and expertise. A stationary VR system and a powerful computer to render sophisticated stereoscopic graphics and compute the biofeedback algorithm are needed. Costly physiological sensors need to be acquired and integrated with the VR system. The measurement is often obtrusive and uncomfortable (e.g., facial masks, belts, electrodes placed on skin, wires running from the sensors to the interface), thus defeating the purpose of relaxation. Consequently, while the effects of a combination of VR and biofeedback seem promising, a broader application is often not feasible due to practical and economic constraints. In an attempt to overcome the aforementioned hindrances, we developed a mobile, allin-one VR-based respiratory biofeedback game to foster diaphragmatic breathing. The game is designed as an intuitive and portable breathing training without the need for external measurement units. We combined existing evidence and best-practice approaches in biofeedback and gamification with mobile VR and a novel built-in breathtracking approach. The present paper gives an overview of the developed VR game and provides an empirical evaluation of its feasibility and effects.

\subsection{Development of a mobile VR-based respiratory biofeedback game}

To offer an immersive and effective, yet accessible and easyto-use biofeedback training, the VR game integrates several components. First, the game is designed and programmed to run on an all-in-one VR headset. This implies that the headset itself contains a central processing unit and a graphics processing unit as well as a battery and all tracking sensors, lest any external computer, sensor or wire is needed. Modern all-in-one headsets feature positional and rotational head tracking with six degrees of freedom (three rotational axes, three translational axes). Sophisticated low-latency head tracking ensures a familiar experience that resembles the real-world sensory experience, which leads to a high degree of immersion and creates a sense of presence (Cummings and Bailenson 2016; Riches et al. 2019). Importantly, those contemporary headsets also offer the same degree of hand tracking via wireless controllers. All of this ensures that our game runs on a portable and affordable headset without sacrificing fidelity, which is crucial in the context of effective biofeedback.

Second, the game puts the user in an immersive and relaxing environment meant to provide a break from 
day-to-day routines. Since mobile graphics rendering limits the artistic possibilities of the application, the virtual environment is designed to require a low amount of rendering power on the one hand, but still bear a restorative potential on the other hand. Additionally, in the context of relaxation training, a stylized fictional environment can offer a novel learning setting unbiased by potentially negative real-world experiences. As such, the game features stylized low-polygon 3D-models and mild but bright color schemes. The models represent typical elements of nature like trees, grass, flowers and rocks. While going for a stylized approach to target mobile graphics rendering and provide a break from well-known everyday surroundings, we still chose associations to elements of nature, because even virtual nature scenery has been shown to restore relaxation and attentional resources by providing a feeling of being away through fascination, extent and compatibility (see meta-analyses on the Attention Restoration Theory, Bowler et al. 2010; McMahan and Estes 2015; Ohly et al. 2016; in the context of VR biofeedback, see also Blum et al. 2019; Rockstroh et al. 2019, 2020). The game consists of two types of landscape scenery with three levels each (see Fig. 1). The first landscape depicts a stylized hillside with rolling hills, large trees and different types of flowers and grass in a blue-to-green color scheme with bright yellow highlights. The second landscape depicts a stylized rocky bay with large cliffs, swaying trees and seaweed in a warm yellow-to-orange color scheme with bright green highlights. We chose two different landscape types with two different color schemes to account for potential preferences among users as well as to offer variety.

Third, in contrast to existing respiratory biofeedback approaches, we avoided the use of external sensors (e.g., nostril sensors, facial masks, stretch sensors). Instead, we developed an algorithm that utilizes the VR hand controller placed on the user's abdomen to detect the respirationinduced abdominal movements in real time without the need for external devices or systems. The controller tracking makes it possible to determine the position of the controller with great precision. With our approach, the user holds one controller in their hand and gently places it on the abdomen. The abdominal movements are transferred to the controller. Thus, the respiration-induced movements of the abdomen can be approximated by the relative positional changes of the controller over time. A forward movement of the controller relative to the abdomen signals expansion of the abdomen (inhalation). A backward movement of the controller relative to the abdomen signals contraction of the abdomen (exhalation). Any positional changes that are not perpendicular to the abdomen as well as any rotational changes of the controller represent movement artifacts and do not signal diaphragmatic breathing. With the help of this algorithm, the positional controller data provides information about the user's diaphragmatic breathing state in real time (for a detailed description and laboratory examination see Blum et al. 2020).

Fourth, the virtual environment and the respiratory feedback do not stand side-by-side, but are integrated with one another in a twofold approach that follows recent empirical evidence: The user's diaphragmatic breathing is (a) reflected in the virtual environment itself (cf. Blum et al. 2019; Rockstroh et al. 2020) and (b) used to control the game progress via gamified breath-based locomotion (cf. van Rooij et al. 2016).

For the environmental stimuli-related feedback, both inhalation and exhalation are reflected in temporary changes in the virtual environment (e.g., slight color changes, particles emitting from blossoms, growing grass). The feedback elements are embedded into features of the virtual environment so that the user can observe the restorative surroundings and simultaneously receives feedback as regards their current breathing at any time. As mentioned above, directly embedding biofeedback stimuli into a virtual environment has been found to increase the salience and (a)

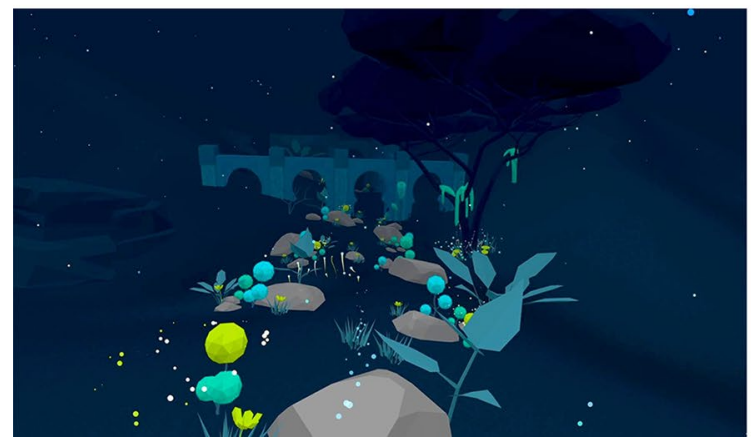

(b)

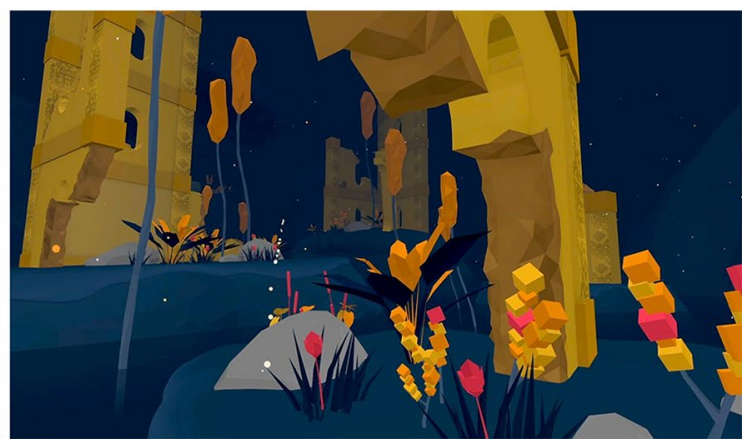

Fig. 1 Screenshots of the two types of virtual environments. The first three levels resemble the left screenshot; the last three levels resemble the right screenshot. Note that the screenshots appear darker than the in-game view with the VR headset 
attractiveness of the feedback, which can foster motivation and focus (cf. Blum et al. 2019; Rockstroh et al. 2019) as well as successful reinforcement learning (cf. Gaume et al. 2016; Sherlin et al. 2011). In turn, the possibility of conscious manipulation can foster the engagement with the virtual environment.

In addition to the environmental feedback, the user is meant to actively use diaphragmatic breathing to move through the virtual environment (cf. van Rooij et al. 2016). Via conscious inhalation and exhalation, the user slowly and gently progresses each of the six levels of the virtual world along a predefined path visually represented by emitting particles along a line. We decided to lock player movement to this path to reduce the required attentional capacity directed to decision making in approaches with free and self-guided navigation. With the fixed-path approach, those attentional resources are conserved and can be allocated to diaphragmatic breathing. The player follows the path using a breath-induced locomotion mechanic that comprises three parts: First, there is a subtle constant force, constantly pulling the player forward along the path at a very slow baseline velocity. This is done to prevent instances of a complete stop or restart, which can create a demotivating impression of being stuck and can increase the risk of simulator sickness. Second, while inhaling, a counter variable is increased (up to a maximum of $4 \mathrm{~s}$ ), which is represented by increasing visibility of the path (greater emission of particles with longer lifetime). Third, while exhaling, an additional forward force is applied, whose strength depends on the aforementioned counter variable. There is no upper limit for the exhalation duration. However, visibility of the path is reduced while exhaling and reaches its low limit after $6 \mathrm{~s}$. Consequently, slow inhales ( $\geq 4 \mathrm{~s}$ ) followed by slow exhales (preferably $6 \mathrm{~s}$; exhale-inhale ratio of 1.5) lead to optimal forward force and thus velocity which create a consistent and even traveling experience. With this breathing-based locomotion approach, conscious diaphragmatic breathing is mandatory to succeed in the game (besides the opportunity to alter the environment). Without or with improper diaphragmatic breathing, the user cannot move forward to explore the world. Thus, we build on the user's intrinsic curiosity to provide an intuitive and rewarding way to guide the user's attention toward diaphragmatic breathing.

We are aware that, while providing a presumably intuitive and engaging biofeedback approach, such artificial locomotion in VR (i.e., movement in the virtual world without corresponding movement in the real world) may increase the risk of simulator sickness, which would defeat the purpose of a tranquil breathing training. Nevertheless, given its intuitive nature and the inherent motivational boost in exploring new surroundings, we chose to stick to the locomotion mechanic. Instead of changing it, we implemented a set of potential remedies. To begin with, we kept the overall locomotion speed to a minimum, taking the form of a soft floating instead of a quick flying. Furthermore, a recent study (Keshavarz and Hecht 2014) hinted at relaxing and pleasant ambience music as a potential countermeasure against visually induced simulator sickness, which we therefore included in the experience. Lastly, recent research has suggested a connection between diaphragmatic breathing and reduced simulator sickness (Russell et al. 2014; Stromberg et al. 2015). Slower breathing rates and a focus on diaphragmatic breathing during sicknessinducing simulation decreased the probability and severity of symptoms. Since the goal of our game lies on practicing conscious diaphragmatic breathing and locomotion is almost exclusively triggered by breathing actions, we expect the aforementioned mechanism to apply to our game.

\subsection{Study and hypotheses}

To investigate the efficacy of the developed game, we conducted a longitudinal intervention study. This study was a follow-up of a previous study in which we empirically investigated the technical feasibility of the breath tracking algorithm in a non-game single session laboratory setting (Blum et al. 2020). Over the course of six training sessions, the present study was meant to evaluate the feasibility of the VR-based respiratory biofeedback game as a training program to foster diaphragmatic breathing. To begin with, we exploratively analyzed general user experience aspects (dropout, liking, ease-of-use, perceived usefulness, simulator sickness) to check whether the game is feasible from a practicable stance.

In a pursuit of evidence as to the effectiveness of the training, we focused on indicators of improved breathing. We investigated both (a) objective improvements in diaphragmatic breathing over the course of the training sessions and (b) subjective impressions regarding diaphragmatic breathing, including perceived ease of performing diaphragmatic breathing over the course of the sessions, as well as perceived breath awareness, that is, how much the participants felt the training as a whole increased their awareness of their own breath:

$H_{1 \mathrm{a}}$ The training improves diaphragmatic breathing over the course of the six sessions.

$H_{1 \mathrm{~b}}$ The training improves the ease of performing diaphragmatic breathing over the course of the six sessions.

$H_{1 \mathrm{c}} \quad$ The training increases the perceived breath awareness. 
In a second step, we investigated whether the training would exert a measurable influence on the participants' overall mental health and well-being. As outlined above, diaphragmatic breathing is used as a key relaxation technique in many stress reduction treatments and has been shown to reduce stress and increase mental health (Hopper et al. 2019; Perciavalle et al. 2017; Zaccaro et al. 2018). Consequently, we investigated the immediate effect of the training sessions of our respiration-based game on participants' relaxation and a potentially more distal effect of the training as a whole on two aspects of mental health, namely perceived stress and symptoms of burnout:

$H_{2 a}$ The training sessions improve participants' momentary relaxation.

$H_{2 \mathrm{~b}} \quad$ The training reduces the perceived stress.

$H_{2 \mathrm{c}}$ The training reduces symptoms of burnout.

Lastly, we tested whether the game would be beneficial in terms of participants' beliefs in their ability to cope with different stressors (i.e., relaxation-related self-efficacy). As mentioned above, the immediate and engaging nature of biofeedback can lead to an increase in self-efficacy because success in exhibiting a healthy behavior or skill is immediately observable. In the present case, finishing the game signals the participants that they have successfully performed diaphragmatic breathing:

$H_{3}$ The training increases the relaxation-related self-efficacy.

\section{Method}

\subsection{Participants and design}

In total, 45 healthy undergraduate participants ( 29 women) took part in the study. Age ranged from 19 to 52 with an average of 22.9 years $(S D=5.4)$. None of the participants had any prior experience with respiratory biofeedback in VR. Participants were recruited via social media as well as a local participant database. Undergraduates received course credit for participation. There was no monetary incentive. All participants gave written informed consent prior to the study.

The study was conducted in our department's VR laboratory using a longitudinal within-subjects design. All participants took part in two assessments prior to the training $\left(t_{0}\right.$ and $t_{1}$; one week apart) to establish untreated baselines, followed by six training sessions in VR over the course of one week and one post-assessment $\left(t_{2}\right.$; after the training). In addition to the control phase between $t_{0}$ and $t_{1}$, we scheduled the participants' individual start of the study at different time points over the course of three months to control for external and seasonal influences.

With each training session, we assessed liking, simulator sickness, objective respiration-induced abdominal movement and perceived ease of performing diaphragmatic breathing. Furthermore, we captured changes in relaxation between the beginning and the end of each training session. At the three main assessments $t_{0}, t_{1}$ and $t_{2}$, we measured perceived breath awareness, perceived stress, symptoms of burnout and relaxation-related self-efficacy. Perceived usefulness of the training and perceived ease of use were assessed at $t_{2}$. The entire experiment was computerized and therefore minimized interaction with the experimenter. After the final assessment, participants were debriefed.

\subsection{Instruments}

\subsubsection{User experience}

Liking was assessed after each training session with one item: "How would you rate today's session?" (from 1 = very bad to $5=$ very good on a Likert scale). Simulator sickness was assessed after each training session with one item: "Did you experience any sickness or nausea during the session?" (from $1=$ did not experience any sickness or nausea to $5=$ experienced sickness or nausea very often). Perceived usefulness of the training as a whole was assessed with two items, one regarding the benefit to diaphragmatic breathing ("The game helped me learn diaphragmatic breathing" on a visual analogue scale [VAS] ranging from $0=$ not at all to $1=$ very much) and the other item regarding the benefit for relaxation ("The game helped me relax" on a VAS ranging from $0=$ not at all to $1=$ very much). Perceived ease of use was assessed via the user friendliness subscale of the Technology Usage Inventory (TUI-BEN; Kothgassner et al. 2012). The scale comprises three items measuring the ease of using a certain technology (in our case, the game) with Likert scales from $1=$ not applicable at all to $7=$ completely applicable (higher ratings indicate greater ease of use).

\subsubsection{Diaphragmatic breathing}

To assess respiration-induced abdominal movements and derive objective breath-related parameters, we recorded every change in the current respiratory status (inhalation movement, exhalation movement, movement artifacts or no movement) as classified by the breath-tracking algorithm based on positional changes of the VR controller during training. For each session, we computed the relative 
share (percentage of the total training session duration) of inhalation movements, exhalation movements and movement artifacts (i.e., controller movement unrelated to breathing) as well as the mean durations of individual inhalation and exhalation movements.

Perceived ease of performing diaphragmatic breathing was assessed via a single item ("How easy was it to perform diaphragmatic breathing?" from $1=$ very difficult to $5=$ very easy). Perceived breath awareness was measured via a selfconstructed scale consisting of eight items. Participants were asked to rate how much each item described their current breath awareness on a Likert scale from $1=$ not at all to $7=$ completely. A sample item is: "I consciously notice most of my breaths." For the entire scale, see "Appendix."

\subsubsection{Mental health}

Momentary relaxation was assessed after each session with a single item: "How relaxed do you feel right now?" (from $1=$ not relaxed at all to $5=$ very relaxed). Perceived stress was measured with the 10-item Perceived Stress Scale (PSS10; Cohen et al. 1983), which asks participants to rate the frequency of stress appraisals defined by unpredictability, uncontrollability and overload in a certain time span on a scale ranging from $0=$ never to $4=$ very often (sample item: "In the last week, how often have you felt nervous and stressed?"). Burnout was measured via the 6-item personal burnout subscale and the 7-item work-related burnout subscale of the Copenhagen Burnout Inventory (CBI; Kristensen et al. 2005). The CBI asks participants to rate their perceptions of strain $(0=$ never/almost never, $25=$ seldom, $50=$ sometimes, $75=$ often, $100=$ very often; sample item: "How often are you emotionally exhausted?").
Our training did not tackle work-related burnout and consequently, we did not expect any related changes in this outcome. Nevertheless, we included the subscale in the analysis as an indicator of a potential halo effect of the training on self-report measures unrelated to the training goal.

\subsubsection{Self-efficacy}

Relaxation-related self-efficacy was measured via a 10-item scale used by Blum and colleagues (2019). With each item, participants are asked to indicate to what extent they are confident to perform the stated action on a VAS from $0=$ not confident at all to $1=$ completely confident (sample item: "How confident are you right now that you can control your worries and fears, even when you are stressed out?").

\subsection{Procedure}

The study consisted of three assessments $\left(t_{0}, t_{1}, t_{2}\right)$ and six training sessions (see Fig. 2), all of which were conducted on-site at the VR laboratory to guarantee a standardized environment. To deliver the VR training, we used an Oculus Quest VR headset. During each assessment, participants filled in the perceived breath awareness scale, the PSS-10, the CBI and the relaxation-related self-efficacy scale via a dedicated PC-based application. After the first assessment, participants were handed a leaflet consisting of a hands-on explanation of the diaphragmatic breathing technique as well as an introduction to the upcoming game, which they were asked to read before the second assessment. After the second assessment, participants immediately proceeded to the first training session. In this session, a female experimenter assisted the participants with the handling of

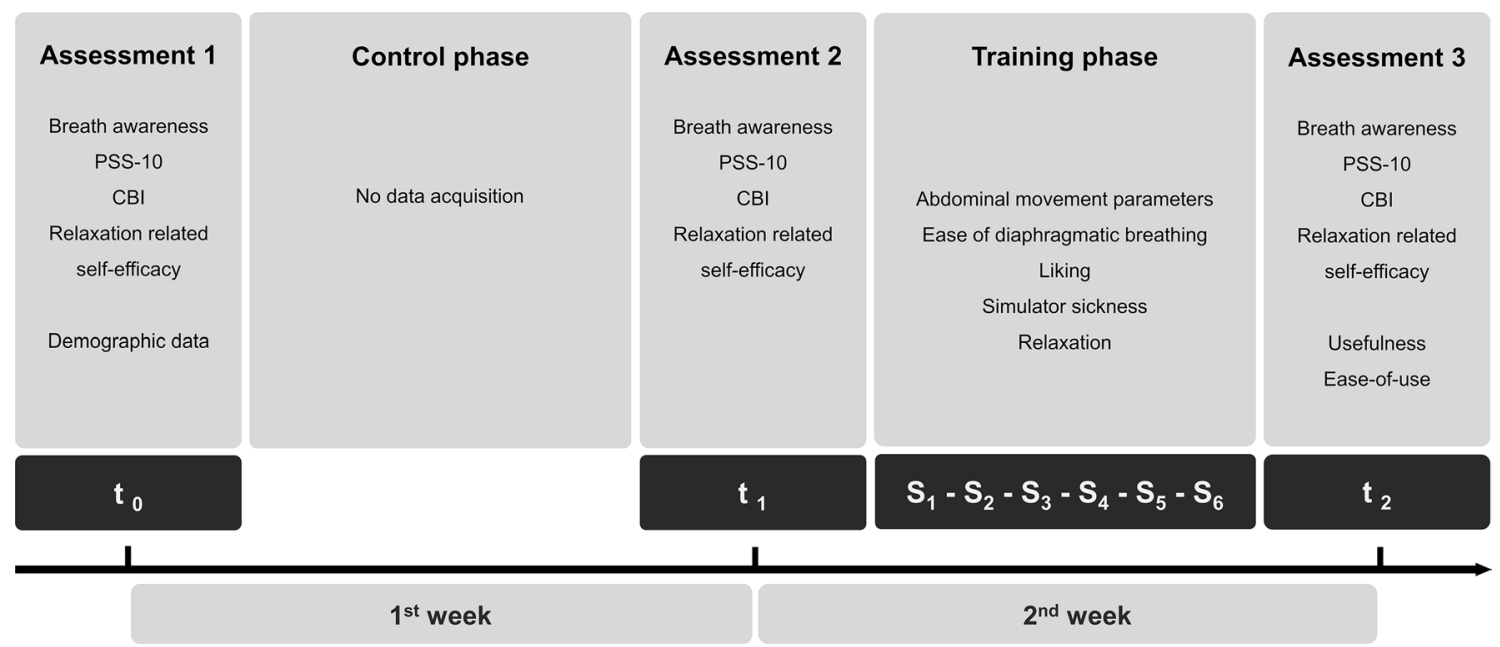

Fig. 2 Timeline of the study. $\mathrm{t}=$ measurement time point, $\mathrm{S}=$ training session 
the VR headset and the correct positioning of the controller utilized for breath tracking. Subsequent sessions were completed autonomously by the participants without further assistance. The first training session included an interactive tutorial integrated into the first level of the game in which the desired breathing technique was rehearsed and the locomotion mechanics were explained. During all sessions, participants were seated in a swivel chair, which supported effortless rotation and visual exploration in the virtual world. Before and after each session, participants indicated their current relaxation. After each session, participants indicated their liking of the session, their perceived ease of performing diaphragmatic breathing and whether they experienced simulator sickness. These ratings were embedded into the virtual reality application in a dedicated neutral menu scene. In each training session, participants completed one level of the game, which took approximately $8 \mathrm{~min}$ on average $(M=463 \mathrm{~s}, S D=83 \mathrm{~s})$. In sum, the whole game took approximately $46 \mathrm{~min}(M=2777 \mathrm{~s}, S D=500 \mathrm{~s})$. In the last assessment $\left(t_{2}\right)$, participants rated the perceived usefulness and ease of use of the training as a whole.

\section{Results}

\subsection{User experience}

Mean values were satisfactory across liking, simulator sickness, perceived usefulness and ease of use. While there are no norm values for the scales and items we used, the fact that all means are above the middle of the respective scale points toward a satisfying user experience. The fact that no participant dropped out of the training underlines the pleasant user experience.

Participants appraised the training sessions positively $(M=4.34, S D=0.47$, scale from 1 to 5$)$ and reported low values of simulator sickness $(M=1.40, S D=0.40$, scale from 1 to 5). There were differences in liking among the sessions, $F(5,220)=4.773, p<.001, \eta_{\mathrm{p}}{ }^{2}=.098$, with the first

Table 1 Liking and simulator sickness across the six training sessions

\begin{tabular}{lll}
\hline Session & Liking $M(S D)$ & $\begin{array}{l}\text { Simulator } \\
\text { sickness } M \\
(S D)\end{array}$ \\
\hline 1 & $4.64(0.57)$ & $1.49(0.73)$ \\
2 & $4.22(0.80)$ & $1.62(1.11)$ \\
3 & $4.20(0.82)$ & $1.33(0.64)$ \\
4 & $4.22(0.88)$ & $1.33(0.77)$ \\
5 & $4.18(0.68)$ & $1.36(0.91)$ \\
6 & $4.56(0.69)$ & $1.29(0.70)$ \\
\hline
\end{tabular}

Liking and simulator sickness were assessed on Likert scales ranging from 1 to 5 and last session scoring higher than the other four sessions. There were no differences in simulator sickness among the sessions, $F(5,220)=1.158, p=.331$. Table 1 shows liking and simulator sickness for the individual training sessions.

Moreover, participants felt that the game had helped them learn diaphragmatic breathing $(M=0.77, S D=0.21$, scale from 0 to 1$)$ and relax $(M=0.80, S D=0.16$, scale from 0 to 1). As regards ease of use, participants ratings on the TUI-BEN scale (Cronbach's $\alpha=.90)$ were high $(M=20.22$, $S D=1.74$; maximum scale value: 21 ).

\subsection{Objective breathing parameters}

Prior to the analyses, we checked the abdominal movement data against controller movement artifacts. Across all sessions, a small fraction of the entire breathing data time series was classified as artifacts $(M=2.8 \%, S D=1.8 \%)$. We excluded these artifacts from the computation of the relative shares of inhalation and exhalation movements to allow for clearer interpretation and an unbiased comparison among the training sessions.

To assess the effect of the training on respiration-induced abdominal movement as an indicator for diaphragmatic breathing $\left(H_{1 \mathrm{a}}\right)$, we analyzed both (1) the relative amount of involving the diaphragm during each session and (2) the mean duration of diaphragm expansion (inhalation) or contraction (exhalation) during individual breaths.

First, we performed separate repeated-measures ANOVAs (6 individual sessions as within-subjects measurement points) for the relative share (in \%) of both inhalation movements and exhalation movements (Fig. 3). The ANOVA on the relative share of inhalation movements revealed an effect of the training over time, $F(3.171$, $139.536)=11.108, p<.001, \eta_{\mathrm{p}}{ }^{2}=.202$, with the relative inhalation share increasing over the course of the training. Difference contrast analyses showed that the relative share

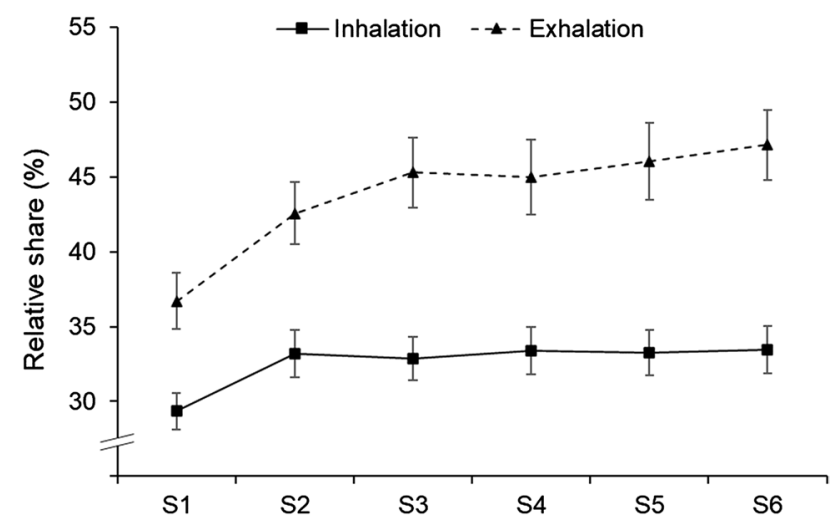

Fig. 3 Relative share (in \%) of inhalation and exhalation movements by training session. Error bars represent $95 \%$ confidence intervals (CI) 
of inhalation for each session (except for Session 5 with $p=.091$ ) was significantly higher compared to the average of all previous sessions (all $p \leq .036$, all $\eta_{\mathrm{p}}{ }^{2} \geq .096$ ), thus corroborating the trend.

The ANOVA on the relative share of exhalation movements revealed an effect of the training over time, $F(3.840,168.977)=29.028, p<.001, \eta_{\mathrm{p}}{ }^{2}=.397$, with the relative exhalation share increasing over the course of the training. Difference contrast analyses showed that the relative share of exhalation movements for each session was significantly higher compared to the average of all previous sessions (all $p \leq .001$, all $\eta_{\mathrm{p}}^{2} \geq .266$ ), thus corroborating the trend.

Next, we conducted two separate repeated-measures ANOVAs (6 individual sessions as within-subjects measurement points) on the mean duration of inhalation movements and exhalation movements (see Fig. 4). The ANOVA on the mean duration of inhalation movements revealed an effect of the training over time, $F(3.688$, $162.282)=17.428, p<.001, \eta_{\mathrm{p}}^{2}=.284$, with the mean duration increasing over the course of the training. In line with that, difference contrast analyses showed that the mean duration of inhalation movements for each session was significantly higher compared to the average of all previous sessions (all $p \leq .005$, all $\eta_{\mathrm{p}}^{2} \geq .163$ ).

The ANOVA on the mean duration of exhalation movements revealed an effect of the training over time, $F(5$, $220)=19.383, p<.001, \eta_{\mathrm{p}}{ }^{2}=.306$, with the mean duration increasing over the course of the training. In line with that, difference contrast analyses showed that the mean duration of exhalation movements for each session was higher than the average of all previous sessions (all $p \leq .001$, all $\eta_{\mathrm{p}}^{2} \geq .269$ ).

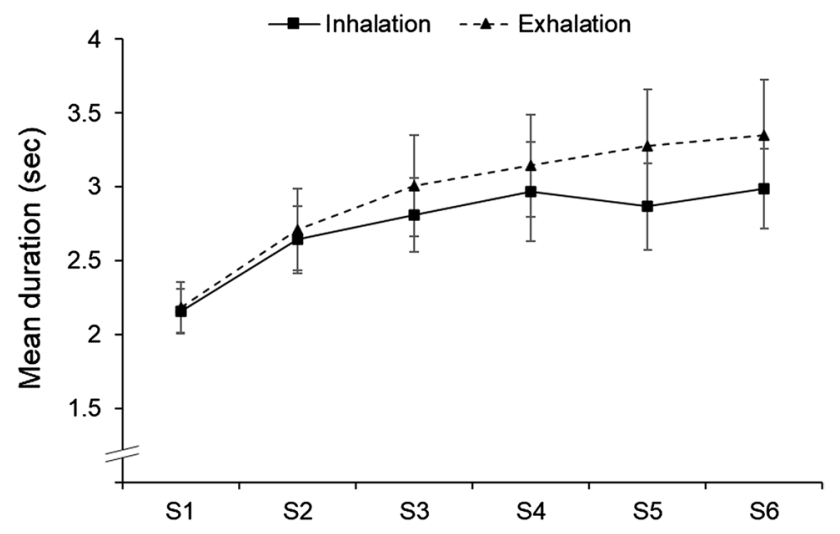

Fig. 4 Mean duration (in sec) of inhalation and exhalation movements by training session. Error bars represent $95 \%$ CI

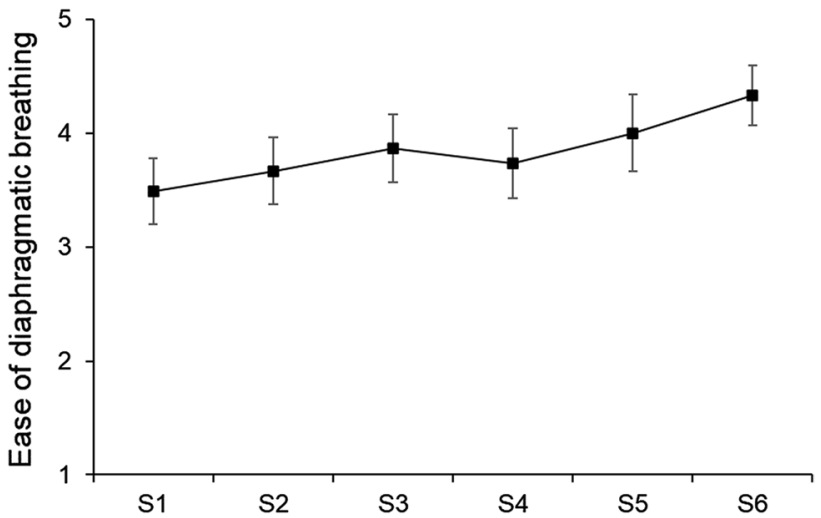

Fig. 5 Perceived ease of diaphragmatic breathing (scale ranging from 1 to 5 , higher values representing greater ease) by training session. Error bars represent 95\% CI

\subsection{Perceived ease of performing diaphragmatic breathing}

To assess the effect of the training on participants' perceived ease of performing diaphragmatic breathing $\left(H_{1 \mathrm{~b}}\right)$, we conducted a repeated-measures ANOVA (6 individual sessions as within-subjects measurement points, see Fig. 5). The analysis revealed an effect of the training over time, $F(5,220)=5.900, p<.001, \eta_{\mathrm{p}}{ }^{2}=.118$. Difference contrast analyses showed that the perceived ease for Sessions 3, 5 and 6 was higher than the average of all previous sessions (all $p \leq .034$ all $1 \eta_{\mathrm{p}}^{2} \geq .098$ ), while there were no differences between Sessions 1 and $2(p=.307)$ and Session 4 compared to all previous sessions $(p=.675)$. Taken together, this establishes a positive trend.

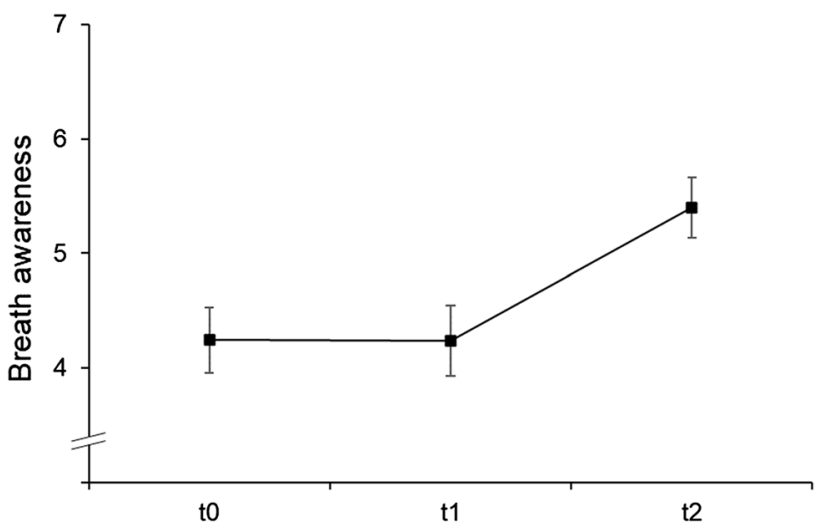

Fig. 6 Perceived breath awareness by measurement point. Error bars represent $95 \% \mathrm{CI}$ 


\subsection{Perceived breath awareness}

The self-constructed scale to assess perceived breath awareness showed a Cronbach's alpha ranging from $\alpha=.77$ $\left(t_{0}\right)$ to $\alpha=.87\left(t_{2}\right)$. To investigate whether the training in whole had an effect on perceived breath awareness $\left(H_{1 c}\right)$, we conducted a repeated-measures ANOVA on the perceived breath awareness scale $\left(t_{0}, t_{1}\right.$ and $t_{2}$ as within-subjects measurement points, Fig. 6). There was an effect of the training, $F(1.644,72.335)=54.941, p<.001, \eta_{\mathrm{p}}{ }^{2}=.555$. Custom contrasts showed that perceived breath awareness did not differ between $t_{0}$ and $t_{1}(p=.953)$ but was higher after the training at $t_{2}$ compared to $t_{0}$ and $t_{1}$ combined $\left(p<.001, \eta_{\mathrm{p}}^{2}=.631\right)$.

\subsection{Relaxation}

To assess an immediate effect of the individual training sessions on participants' relaxation $\left(H_{2 \mathrm{a}}\right)$, we conducted a repeated-measures ANOVA with the factors Training Session (6 individual sessions as within-subjects measurement points) and Time ( 2 within-subjects levels: pre-session, post-session). The ANOVA on relaxation revealed an effect of Time, $F(1,44)=140.315, p<.001$, $\eta_{\mathrm{p}}^{2}=.761$, with higher relaxation ratings post-session $(M=4.31, S D=0.48)$ compared to pre-session $(M=3.31$, $S D=0.67)$. There was no effect of Training Session, $F(3.722,163.758)=1.727, p=.151$, and no Time $\times$ Training Session interaction, $F(5,220)=0.504, p=.773$, indicating that the training sessions improved momentary relaxation equally well.

\subsection{Perceived stress}

The PSS-10 showed a Cronbach's alpha ranging from $\alpha=.82$ $\left(t_{0}\right)$ to $\alpha=.93\left(t_{2}\right)$. To investigate an effect of the training on perceived stress $\left(H_{2 \mathrm{~b}}\right)$, we conducted a repeated-measures

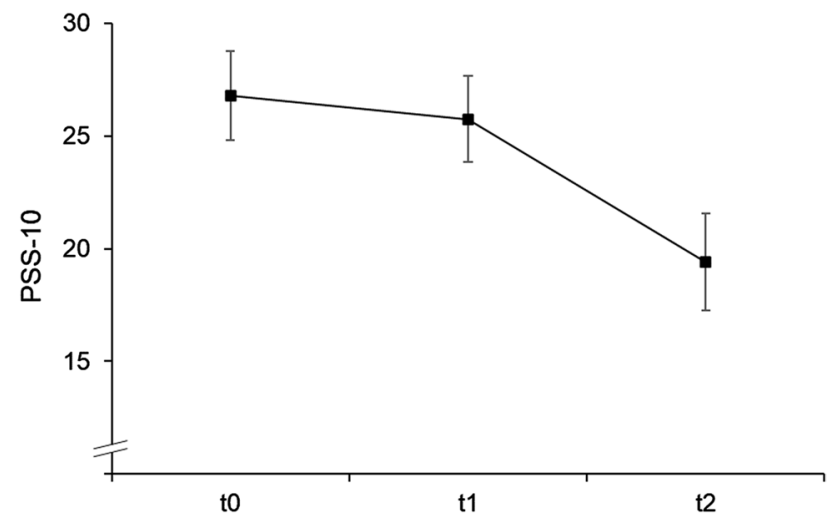

Fig. 7 Perceived stress (PSS-10) by measurement point. Error bars represent 95\% CI
ANOVA on the PSS-10 scores $\left(t_{0}, t_{1}\right.$ and $t_{2}$ as withinsubjects measurement points, Fig. 7). The analysis revealed an effect of the training, $F(1.426,62.736)=31.254, p<.001$, $\eta_{\mathrm{p}}^{2}=.415$. Custom contrasts showed that PSS- 10 scores did not differ between $t_{0}$ and $t_{1}(p=.101)$ but were lower after the training at $t_{2}$ compared to $t_{0}$ and $t_{1}$ combined $(p<.001$, $\left.\eta_{\mathrm{p}}^{2}=.463\right)$.

\subsection{Burnout symptoms}

The CBI showed a Cronbach's alpha with the personal subscale ranging from $\alpha=.78\left(t_{0}\right)$ to $\alpha=.84\left(t_{2}\right)$ and the work-related subscale ranging from $\alpha=.77\left(t_{0}\right)$ to $\alpha=.85$ (both $t_{1}$ and $t_{2}$ ). To assess an effect of the training on burnout symptoms $\left(H_{2 c}\right)$, we conducted two separate repeatedmeasures ANOVA on the personal and work-related CBI scores $\left(t_{0}, t_{1}\right.$ and $t_{2}$ as within-subjects measurement points, see Fig. 8). With the personal burnout scores, the analysis revealed an effect of the training, $F(1.372,60.370)=35.080$, $p<.001, \eta_{\mathrm{p}}^{2}=.444$. Custom contrasts showed that the scores

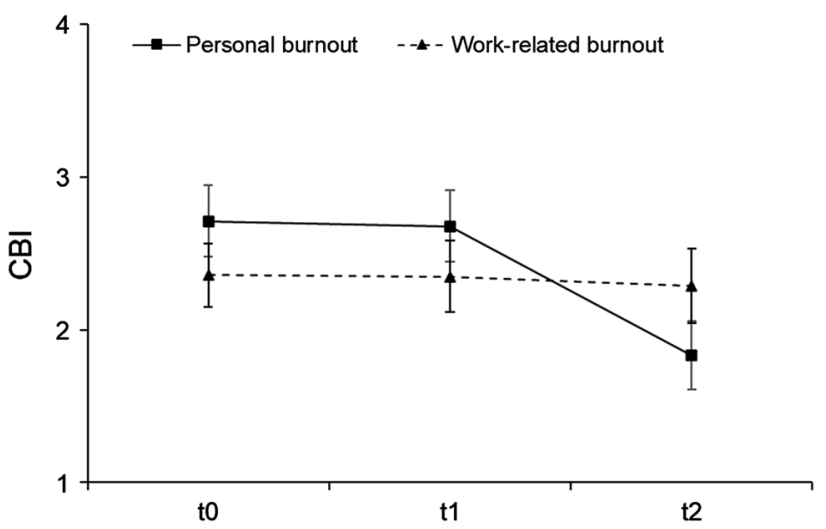

Fig. 8 Personal and work-related burnout (CBI) by measurement point. Error bars represent $95 \%$ CI

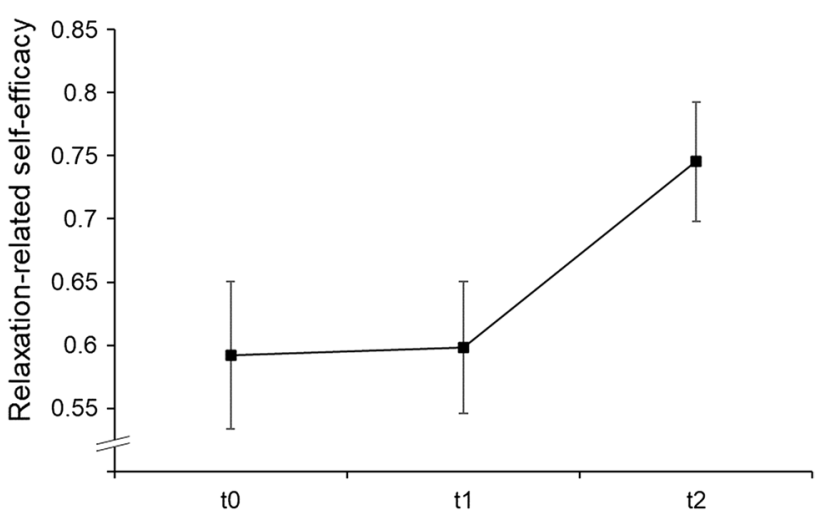

Fig. 9 Relaxation-related self-efficacy by measurement point. Error bars represent $95 \% \mathrm{CI}$ 
on the personal subscale of the CBI did not differ between $t_{0}$ and $t_{1}(p=.626)$, but were lower after the training at $t_{2}$ compared to $t_{0}$ and $t_{1}$ combined $\left(p<.001, \eta_{\mathrm{p}}{ }^{2}=.488\right)$. For the work-related burnout scale, there was no effect of the training, $F(2,88)=0.548, p=.580$.

\subsection{Relaxation-related self-efficacy}

The scale to assess relaxation-related self-efficacy showed a Cronbach's alpha ranging from $\alpha=.91\left(t_{1}\right)$ to $\alpha=.95\left(t_{2}\right)$. To investigate an effect of the training on relaxation-related selfefficacy $\left(H_{3}\right)$, we conducted a repeated-measures ANOVA $\left(t_{0}, t_{1}\right.$ and $t_{2}$ as within-subjects measurement points, Fig. 9) on the scale values. The analysis revealed an effect of the training, $F(1.341,59.017)=32.650, p<.001, \eta_{\mathrm{p}}{ }^{2}=.426$. Custom contrasts showed that relaxation-related self-efficacy did not differ between $t_{0}$ and $t_{1}(p=.612)$ but was higher after the training at $t_{2}$ compared to $t_{0}$ and $t_{1}$ combined $(p<.001$, $\left.\eta_{\mathrm{p}}^{2}=.468\right)$.

\section{Discussion}

The present longitudinal study investigated the feasibility of a novel VR-based respiratory biofeedback game. Besides investigating user experience aspects, we tested the effects of the six-session training on objective parameters of diaphragmatic breathing, the perceived ease of performing diaphragmatic breathing and the perceived breath awareness. Moreover, we explored whether the respiration training influenced momentary relaxation, perceived stress, symptoms of burnout and relaxation-related self-efficacy. Overall, the results provide preliminary evidence as to the practicability and efficacy of the game to train diaphragmatic breathing and improve short-term mental health.

With regard to the aspects of user experience, the study yielded promising results. Over the course of six training sessions and a total duration of approximately $46 \mathrm{~min}$, participants rated the training to be likeable, easy to use and useful while no participant dropped out of the training. As to the reason for the higher liking ratings after the first and last session, we can only speculate. One potential explanation is that the first rating was driven by a novelty effect and the last rating by an effect of having finished the training successfully. Notably though, all liking ratings were high. The fact that the participants perceived the training as useful to learn diaphragmatic breathing is promising because any training application profits if trainees believe in its usefulness. Simulator sickness was not an issue. All participants reported only minor degrees of simulator sickness at most, with most sessions not evoking simulator sickness at all. This is in line with our assumption that the simultaneous occurrence of artificial movement in the game and conscious exhalation reduces the probability of simulator sickness. The comforting surroundings combined with calm ambience music and the low velocity are likely to have had an influence, as well. To sum up, the ratings regarding the overall user experience suggest that the game forms a solid foundation for a breathing training.

As regards the fulfillment of the actual training goalimproving diaphragmatic breathing - the results are positive. The objective abdominal movement data show that the participants gradually increased the involvement of the diaphragm in their breathing over the course of the training. The relative share of diaphragm movement (inhalation and exhalation taken together) in the last session differed from the first session by $14.5 \%$, which equals a $22 \%$ increase from the first to the last session. Moreover, the analyses regarding the mean inhalation and exhalation duration indicate that the participants increased the length of the individual breath cycles, that is, they breathed slower toward the end of the training. During the last session, the relative amount of diaphragm movement (inhalation and exhalation taken together) reached $80.6 \%$ and the mean breathing duration (inhalation and exhalation taken together) was $6.3 \mathrm{~s}$. Given that the recorded abdominal movements are likely to underestimate the true value due to the inherent lag of trendbased algorithms, these numbers indicate training success. When additionally considering that regular diaphragmatic breathing includes short periods of 1-2 s of breath holding in-between cycles, the results suggest that, on average, the participants performed close to $100 \%$ of diaphragmatic breathing with an estimated breath cycle duration of $8 \mathrm{~s}$ (when assuming half a second of breath holding for each inhale and exhale, respectively). This translates to an approximate respiratory rate of 7.5 breaths per minute. Considering that the participants underwent only six brief training sessions, this result is encouraging. Moreover, in the last session, participants reached an exhale-inhale ratio of $1.46(S D=0.40)$, which is close to the desired ratio of 1.5 put forward by our locomotion-based feedback implementation.

Considering the progression of the breathing data from session to session, two observations are worth mentioning. First, the largest improvement can be observed from the first to the second session. This indicates that the first session already helped the participants to a substantial degree. This is interesting because it reflects the self-reported ease of use, that is, the participants quickly grasped the locomotion mechanics, and it suggests that even a single session of an immersive VR-based biofeedback game might help raise the awareness of one's diaphragmatic breathing. Second, it can be observed that the training effect is gradually flattened over the course of the six sessions. It looks as if the participants reached their best possible breathing style already after the sixth session. Based on this preliminary result and considering potential benefits from repeated and 
spaced practice, we suggest an approximate session number of 10-12 with increasing intervals between the sessions.

The training's effectiveness as regards the abdominal movement data is reflected by the participants' perceived impression of being more and more able to perform adequate diaphragmatic breathing. This indicates that the feedback implementation was successful in feeding back the breathing style, and participants were aware of their improved performance. When training behavioral skills like diaphragmatic breathing, the trainee's subjective impression of the training progress is important above and beyond the objective improvements in terms of motivation and compliance. In addition to the increased ease of performing diaphragmatic breathing from session to session, participants reported greater general breath awareness after the completion of the training. Notably, the written explanation of diaphragmatic breathing as provided in the leaflet before the start of the training did not increase awareness. Not surprisingly, the immersive nature of experiencing one's own breath in the VR environment appears to exert a stronger influence on the awareness as compared to written information.

In sum, the objective and subjective breathing data imply a substantial training effect. It should be noted that, despite the promising results, this study only comprises breathing data during the training itself and does not provide evidence as to potential long-term effects as well as the transfer to non-training contexts. Nonetheless, the present study provides promising preliminary evidence and opens up new research avenues that may investigate aspects of training transfer and long-term effects as well as comparisons with traditional breathing trainings.

The present study also considered measures of subjective stress, in the context of both momentary relaxation after individual training sessions $\left(H_{2 \mathrm{a}}\right)$, as well as effects of the training as a whole $\left(H_{2 \mathrm{~b}}\right.$ and $\left.H_{2 \mathrm{c}}\right)$. As regards momentary relaxation, the results underline the impact of the individual training sessions. After each training session, the relaxation was deeper than before the session. When considering the well-established effectiveness of diaphragmatic breathing exercises on momentary stress and well-being, this might not be surprising. Nevertheless, we deem the finding worth noticing because the present VR-based biofeedback game confronted the participants with a set of novel, technology-driven approaches, for instance all-in-one VR, biofeedback and the specific locomotion mechanics. Despite these potential stressors, the focus on experiencing one's own breath in VR seemed to have helped the participants relax. The momentary relaxation effect of individual training sessions also manifested itself in an overall stress reduction outside of and after the entire training. While the assessments $\left(t_{0}, t_{1}, t_{2}\right)$ only span just over two weeks and therefore do not allow for a long-term analysis of perceived stress, the results provide preliminary evidence as to a potential stress-reducing effect of the training. Both perceived stress and symptoms of personal burnout were significantly reduced over the course of the training. Again, this effect can neither be accounted for by a repeated measurement artifact nor a mere effect of psychoeducation because there were no significant differences between $t_{0}$ and $t_{1}$. Notably, there was also no effect of the training on work-related burnout symptoms. This is positive because work-related burnout was not targeted by the training and the lack of an effect reduces the likelihood of a mere haloeffect on the self-report measures in general. As with the breathing data, we consider the results regarding stress reduction through the VR-based respiratory biofeedback game promising, especially given the brief duration, the necessity to adapt to a completely unfamiliar training and the consistently large effect sizes.

Lastly, the study repeatedly captured relaxation-related self-efficacy. Previous research points toward an effect of biofeedback on self-efficacy, while VR-based biofeedback was found to have an even greater effect. The present study corroborates these findings. After the training, participants' general relaxation-related self-efficacy was significantly increased. It appears as though the participants gained confidence in their own stress reduction abilities through the training. This is likely due to the effects on diaphragmatic breathing, specifically in terms of the perceived ease of performing diaphragmatic breathing.

\subsection{Limitations and future research}

Despite the promising findings, the measurement approach and the study design limit the generalizability and specificity of the results. A number of limitations need to be kept in mind when interpreting the results and should be taken into consideration in future studies: One limitation is the lack of a control group, especially the lack of an active control condition. We remedied the lack of an untreated control group, at least in part, by implementing a double pre-measurement to account for potential testing effects. Moreover, we scheduled the start of the training over the course of three months to reduce external and seasonal influences. We therefore decided to forego an untreated control condition and prioritize a greater number of participants that tested the developed game. However, the lack of an active control condition keeps the study from identifying the specific factors that account for the effects we found. Because the developed game comprises several potentially effective factors (VR, restorative environments, breathing exercises, biofeedback), it is impossible to tell which of these factors or their combinations contributed how much to the results. Future research may disentangle the effects of these factors, even though this will not be 
an easy endeavor due to the manifold potential ways of implementing and comparing the factors and the great number of experimental conditions and participants to realize them. To us, the threat of comparing apples with oranges appeared too high to allocate resources to an active control condition in this feasibility study.

The study did not include a long-term assessment of the effects. Again, this was due to economical constraints and should be considered in future investigations. All reported effects, even though some were measured over three measurement points, are to be considered short-term effects. On a similar note, the abdominal movement data were exclusively taken during the training sessions. Further investigations should consider additional pre- and postmeasurements of diaphragmatic breathing to test the transfer to different contexts.

Moreover, the present sample was too homogenous to extrapolate the findings to many kinds of potential trainees. Participants with varying age, affinity to technology, breathing skills, experience and stress levels or even different clinical conditions should be subject to future research.

Besides these research suggestions based on the limitations in this study, future research could explore different session numbers to find the optimal balance between a brief training and sufficient repeated practice, investigate the effects and potential of different kinds of virtual environments and establish the novel breathing algorithm further.

\section{Conclusion}

This study reported on the development and evaluation of a novel VR-based respiratory biofeedback game to foster diaphragmatic breathing. Our approach targeted mobile all-in-one VR and implemented a built-in respiratory biofeedback algorithm utilizing the VR hand controller. A longitudinal evaluation study established the feasibility of the approach and provided evidence for improved diaphragmatic breathing and breath awareness. Furthermore, the brief sixsession training positively influenced relaxation, perceived stress and symptoms of burnout and boosted the participants' relaxation-related self-efficacy. The developed VR-based biofeedback game provides a low-cost, easy-to-use and effective way to train diaphragmatic breathing. Future research is needed to corroborate the findings, compare the training to different approaches and explore long-term effects.
Funding Open Access funding enabled and organized by Projekt DEAL. This research did not receive any grant from funding agencies in the public, commercial or not-for-profit sectors.

\section{Compliance with ethical standards}

Conflict of interest The authors declare that they have no conflict of interest.

Availability of data and material (data transparency) The datasets generated for this study are available on request to the corresponding author.

Ethics statement The present study has been carried out in accordance with the APA Guidelines for Ethical Conduct of Behavioral Projects Involving Human Participants and The Code of Ethics of the World Medical Association (Declaration of Helsinki) for experiments involving humans. Written informed consent was obtained from all participants prior to participation. The privacy rights have been observed carefully. The study was conducted anonymously. There was no deception in any way. The participants were given the opportunity to withdraw their consent at any time and terminate their participation. Ethical review and approval was not required for the study on human participants in accordance with the local legislation and institutional requirements.

Open Access This article is licensed under a Creative Commons Attribution 4.0 International License, which permits use, sharing, adaptation, distribution and reproduction in any medium or format, as long as you give appropriate credit to the original author(s) and the source, provide a link to the Creative Commons licence, and indicate if changes were made. The images or other third party material in this article are included in the article's Creative Commons licence, unless indicated otherwise in a credit line to the material. If material is not included in the article's Creative Commons licence and your intended use is not permitted by statutory regulation or exceeds the permitted use, you will need to obtain permission directly from the copyright holder. To view a copy of this licence, visit http://creativecommons .org/licenses/by/4.0/.

\section{Appendix}

\section{Breath awareness scale}

Please indicate to what extent the following statements apply.

(7-point Likert rating scale; $1=$ not at all, $7=$ completely).

1. I pay attention to my breath in everyday life.

2. I mainly breathe through my belly.

3. I consciously notice most of my breaths.

4. I am good at controlling my breathing.

5. I consciously take the time to take a deep breath.

6. When I take a calm breath, I feel relaxed.

7. I immediately take notice when my breath is quick or restless.

8. I feel the difference between abdominal breathing and chest breathing. 


\section{References}

Berger AM, Davelaar EJ (2018) Frontal alpha oscillations and attentional control: a virtual reality neurofeedback study. Neuroscience 378:189-197. https://doi.org/10.1016/j.neuroscien ce.2017.06.007

Blum J, Rockstroh C, Göritz AS (2019) Heart rate variability biofeedback based on slow-paced breathing with immersive virtual reality nature scenery. Front Psychol 10:2172. https://doi. org/10.3389/fpsyg.2019.02172

Blum J, Rockstroh C, Göritz AS (2020) Development and pilot test of a virtual reality respiratory biofeedback approach. Advance online publication, Applied Psychophysiology and Biofeedback. https:// doi.org/10.1007/s10484-020-09468-x

Bowler DE, Buyung-Ali LM, Knight TM, Pullin AS (2010) A systematic review of evidence for the added benefits to health of exposure to natural environments. BMC Public Health 10:456. https://doi.org/10.1186/1471-2458-10-456

Carl E, Stein AT, Levihn-Coon A, Pogue JR, Rothbaum B, Emmelkamp P, Powers MB (2019) Virtual reality exposure therapy for anxiety and related disorders: A meta-analysis of randomized controlled trials. J Anxiety Disord 61:27-36. https://doi.org/10.1016/j.janxd is. 2018.08 .003

Chandrasiri A, Collett J, Fassbender E, De Foe A (2020) A virtual reality approach to mindfulness skills training. Virtual Real 24:143-149. https://doi.org/10.1007/s10055-019-00380-2

Chen YF, Huang XY, Chien CH, Cheng JF (2017) The effectiveness of diaphragmatic breathing relaxation training for reducing anxiety. Perspect Psychiatr Care 53:329-336. https://doi.org/10.1111/ ppc. 12184

Cohen S, Kamarck T, Mermelstein R (1983) A global measure of perceived stress. J Health Soc Behav 24:386-396. https://doi. org/10.2307/2136404

Cummings JJ, Bailenson JN (2016) How immersive is enough? A metaanalysis of the effect of immersive technology on user presence. Media Psychol 19:272-309. https://doi.org/10.1080/15213 269.2015.1015740

Gaume A, Vialatte A, Mora-Sanchez A, Ramdani C, Vialatte FB (2016) A psychoengineering paradigm for the neurocognitive mechanisms of biofeedback and neurofeedback. Neurosci Biobehav Rev 68:891-910. https://doi.org/10.1016/j.neubi orev.2016.06.012

Giardino ND, Chan L, Borson S (2004) Combined heart rate variability and pulse oximetry biofeedback for chronic obstructive pulmonary disease: Preliminary findings. Appl Psychophysiol Biofeedback 29:121-133. https://doi.org/10.1023/B:APBI.0000026638.64386 .89

Giggins OM, Persson UM, Caulfield B (2013) Biofeedback in rehabilitation. J Neuroeng Rehabil 10:60. https://doi. org/10.1186/1743-0003-10-60

Gradl S, Wirth M, Zillig T, Eskofier BM (2018) Visualization of heart activity in virtual reality: A biofeedback application using wearable sensors. In: Proceedings of the 15th International Conference on Wearable and Implantable Body Sensor Networks (pp. 152-155). New York, NY: IEEE. Doi: https://doi. org/10.1109/BSN.2018.8329681

Gromala D, Tong X, Choo A, Karamnejad M, Shaw CD (2015) The virtual meditative walk: virtual reality therapy for chronic pain management. In: Proceedings of the 33rd Annual ACM conference on human factors in computing systems (pp. 521-524). New York, NY: ACM. Doi: https://doi.org/10.1145/2702123.2702344

Goldberg D, Williams P (1988) A user's guide to the general health questionnaire. NFER-Nelson, Windsor
Hølledig ML, Petersen RK (2018) ZenVR: a biofeedback virtual reality experience for assisting meditation. Unpublished master's thesis. Aalborg University, Copenhagen, Denmark

Hopper SI, Murray SL, Ferrara LR, Singleton JK (2019) Effectiveness of diaphragmatic breathing on physiological and psychological stress in adults: a quantitative systematic review protocol. JBI Database Syst Rev Implementation Rep 9:1855-1876. https://doi. org/10.11124/JBISRIR-2017-003848

Houzangbe S, Christmann O, Gorisse G, Richir S (2019) Effects of voluntary heart rate control on user engagement in virtual reality. In: Proceedings of the 2019 Conference on Virtual Reality and 3D User Interfaces (pp. 982-983). New York, NY: IEEE. Doi: https ://doi.org/10.1109/VR.2019.8797759

Keshavarz B, Hecht H (2014) Pleasant music as a countermeasure against visually induced motion sickness. Appl Ergon 45:521527. https://doi.org/10.1016/j.apergo.2013.07.009

Kristensen TS, Borritz M, Villadsen E, Christensen KB (2005) The copenhagen burnout inventory: A new tool for the assessment of burnout. Work Stress 19:192-207. https://doi.org/10.1080/02678 370500297720

Kosunen I, Salminen M, Järvelä S, Ruonala A, Ravaja N, Jacucci G (2016) RelaWorld: neuroadaptive and immersive virtual reality meditation system. In: Proceedings of the 21 st International Conference on Intelligent User Interfaces (pp. 208-217). New York, NY: ACM. Doi: https://doi.org/10.1145/2856767.2856796

Kothgassner OD, Felnhofer A, Hauk N, Kasthofer E, Gomm J, KryspinExner I (2012) TUI: Technology Usage Inventory. Fragebogen und Manual. FFG, Wien

Ma X, Yue ZQ, Gong ZQ, Zhang H, Duan NY, Shi YT, Wei GX, Li YF (2017) The effect of diaphragmatic breathing on attention, negative affect and stress in healthy adults. Front Psychol 8:874. https://doi.org/10.3389/fpsyg.2017.00874

Mallari B, Spaeth EK, Goh H, Boyd BS (2019) Virtual reality as an analgesic for acute and chronic pain in adults: a systematic review and meta-analysis. J Pain Res 12:2053-2085. https://doi. org/10.2147/JPR.S200498

McMahan EA, Estes D (2015) The effect of contact with natural environments on positive and negative affect: a meta-analysis. J Posit Psychol 10:507-519. https://doi.org/10.1080/17439 760.2014.994224

Navarro-Haro MV, López-del-Hoyo Y, Campos D, Linehan MM, Hoffman HG, García-Palacios A, Garcia-Campayo J (2017) Meditation experts try virtual reality mindfulness: a pilot study evaluation of the feasibility and acceptability of virtual reality to facilitate mindfulness practice in people attending a mindfulness conference. PLoS ONE 12:e0187777. https://doi. org/10.1371/journal.pone.0187777

Ohly H, White MP, Wheeler BW, Bethel A, Ukoumunne OC, Nikolaou V, Garside R (2016) Attention restoration theory: a systematic review of the attention restoration potential of exposure to natural environments. J Toxicol Environ Health 19:305-343. https://doi.org/10.1080/10937404.2016.1196155

Paul M, Garg K (2012) The effect of heart rate variability biofeedback on performance psychology of basketball players. Appl Psychophysiol Biofeedback 37:131-144. https://doi. org/10.1007/s10484-012-9185-2

Perciavalle V, Blandini M, Fecarotta P, Buscemi A, Di Corrado D, Bertolo L, Coco M (2017) The role of deep breathing on stress. Neurol Sci 38:451-458. https://doi.org/10.1007/s1007 2-016-2790-8

Riches S, Elghany S, Garety P, Rus-Calafell M, Valmaggia L (2019) Factors affecting sense of presence in a virtual reality social environment: a qualitative study. Cyberpsychol Behav Soc Netw 22:288-292. https://doi.org/10.1089/cyber.2018.0128

Roche K, Liu S, Siegel S (2019) The effects of virtual reality on mental wellness: a literature review. Mental Health Family Med 
14:811-818. Retrieved from https://www.mhfmjournal.com/pdf/ the-effects-of-virtual-reality-on-mental-wellness-a-literature-revie w.pdf

Rockstroh C, Blum J, Göritz AS (2019) Virtual reality in the application of heart rate variability biofeedback. Int J Hum Comput Stud 130:209-220. https://doi.org/10.1016/j.ijhcs.2019.06.011

Rockstroh C, Blum J, Göritz AS (2020) Combining VR and biofeedback: the effects on presence and perceived restorativeness. J Media Psychol. https://doi.org/10.1027/1864-1105/a000270

Russell MEB, Hoffman B, Stromberg S, Carlson CR (2014) Use of controlled diaphragmatic breathing for the management of motion sickness in a virtual reality environment. Appl Psychophysiol Biofeedback 39:269-277. https://doi.org/10.1007/s1048 4-014-9265-6

Scapin S, Echevarria-Guanilo ME, Junior PRBF, Goncalves N, Rocha PK, Coimbra R (2018) Virtual reality in the treatment of burn patients: a systematic review. Burns 44:1403-1416. https://doi. org/10.1016/j.burns.2017.11.002

Sherlin LH, Arns M, Lubar J, Heinrich H, Kerson C, Strehl U, Sterman MB (2011) Neurofeedback and basic learning theory: implications for research and practice. J Neurother 15:292-304. https://doi. org/10.1080/10874208.2011.623089

Soyka F, Leyrer M, Smallwood J, Ferguson C, Riecke BE, Mohler BJ (2016) Enhancing stress management techniques using virtual reality. In: Proceedings of the ACM Symposium on Applied Perception (pp. 85-88). New York, NY: ACM. Doi: https://doi. org/10.1145/2931002.2931017

Sra M, Xu X, Maes P (2018) Breathvr: Leveraging breathing as a directly controlled interface for virtual reality games. In: Proceedings of the 2018 CHI Conference on Human Factors in Computing Systems (pp. 1-12). New York, NY: ACM. Doi: https ://doi.org/10.1145/3173574.3173914

Stromberg SE, Russell ME, Carlson CR (2015) Diaphragmatic breathing and its effectiveness for the management of motion sickness. Aerosp Med Hum Perform 86:452-457. https://doi. org/10.3357/AMHP.4152.2015

Subbalakshmi NK, Adhikari P, Jeganathan PS (2014) Comparative study on cardiac autonomic modulation during deep breathing test and diaphragmatic breathing in type 2 diabetes and healthy subjects. J Diabetes Investig 5:456-463. https://doi.org/10.1111/ jdi. 12163

Teufel M, Stephan K, Kowalski A, Käsberger S, Enck P, Zipfel S, Giel KE (2013) Impact of biofeedback on self-efficacy and stress reduction in obesity: a randomized controlled pilot study. Appl Psychophysiol Biofeedback 38:177-184. https://doi.org/10.1007/ s10484-013-9223-8

Tinga AM, Nyklíček I, Jansen MP, de Back TT, Louwerse MM (2019) Respiratory biofeedback does not facilitate lowering arousal in meditation through virtual reality. Appl Psychophysiol Biofeedback 44:51-59. https://doi.org/10.1007/s 1048 4-018-9421-5

van Rooij M, Lobel A, Harris O, Smit N, Granic I (2016) DEEP: A biofeedback virtual reality game for children at-risk for anxiety. In: Proceedings of the $2016 \mathrm{CHI}$ Conference Extended Abstracts on Human Factors in Computing Systems (pp. 1989-1997). New York, NY: ACM. Doi: https://doi.org/10.1145/2851581.2892452

Vaschillo EG, Vaschillo B, Lehrer PM (2006) Characteristics of resonance in heart rate variability stimulated by biofeedback. Appl Psychophysiol Biofeedback 31:129-142. https://doi.org/10.1007/ s10484-006-9009-3

Weerdmeester J, van Rooij M, Harris O, Smit N, Engels RC, Granic I (2017) Exploring the role of self-efficacy in biofeedback video games. In: Extended abstracts publication of the annual symposium on computer-human interaction in play (pp. 453461). New York, NY: ACM. Doi: https://doi.org/10.1145/31308 59.3131299

Yasuma F, Hayano JI (2004) Respiratory sinus arrhythmia: Why does the heartbeat synchronize with respiratory rhythm? Chest 125:683-690. https://doi.org/10.1378/chest.125.2.683

Yu B, Funk M, Hu J, Wang Q, Feijs L (2018) Biofeedback for everyday stress management: a systematic review. Front in ICT 5:23. https ://doi.org/10.3389/fict.2018.00023

Zaccaro A, Piarulli A, Laurino M, Garbella E, Menicucci D, Neri B, Gemignani A (2018) How breath-control can change your life: a systematic review on psycho-physiological correlates of slow breathing. Front Hum Neurosci 12:353. https://doi.org/10.3389/ fnhum.2018.00353

Publisher's Note Springer Nature remains neutral with regard to jurisdictional claims in published maps and institutional affiliations. 